\title{
Leptin is not associated independently with hypertension in Japanese-Brazilian women
}

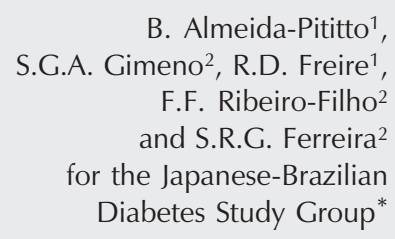

B. Almeida-Pititto ${ }^{1}$, S.G.A. Gimeno², R.D. Freire', F.F. Ribeiro-Filho ${ }^{2}$ and S.R.G. Ferreira ${ }^{2}$ for the Japanese-Brazilian Diabetes Study Group*

\author{
${ }^{1}$ Disciplina de Endocrinologia, Departamento de Medicina Interna, \\ ${ }^{2}$ Departamento de Medicina Preventiva, Escola Paulista de Medicina, \\ Universidade Federal de São Paulo, São Paulo, SP, Brasil
}

\begin{abstract}
Correspondence

B. Almeida-Pititto

Rua Tutoia, 587, Apto. 22

04023-062 São Paulo, SP

Brasil

Fax: +55-11-5549-5159

E-mail: almeida.bi@uol.com.br

Research supported by FAPESP.

Received January 21, 2005

Accepted August 30, 2005

We evaluated the relationship of leptin with hypertension adjusted for body mass index (BMI) and/or waist circumference in a population of Japanese-Brazilian women aged $\geq 30$ years with centrally distributed adiposity. After excluding diabetic subjects, the study subjects - who participated in a population-based study on the prevalence of metabolic syndrome - showed prevalence rates of obesity (BMI $\geq 25 \mathrm{~kg} / \mathrm{m}^{2}$ ) and central adiposity (waist $\geq 80 \mathrm{~cm}$ ) of 32.0 and $37.8 \%$, respectively. The hypertensive group $(\mathrm{N}=162)$ was older, had higher BMI $(24.9 \pm$ 4.2 vs $\left.23.3 \pm 3.4 \mathrm{~kg} / \mathrm{m}^{2}, \mathrm{P}<0.001\right)$, waist circumference $(81.1 \pm 10.1$ vs $76.3 \pm 8.2 \mathrm{~cm}, \mathrm{P}<0.001)$ and insulin levels $(8.0 \pm 6.2$ vs $7.1 \pm 4.9$ $\mu \mathrm{U} / \mathrm{mL}, \mathrm{P}<0.05)$ than the normotensive group $(\mathrm{N}=322)$ and showed an unfavorable metabolic profile (higher 2-h plasma glucose, Creactive protein and non-HDL cholesterol levels). Leptin did not differ between groups $(8.2 \pm 6.8$ vs $7.2 \pm 6.6 \mathrm{ng} / \mathrm{mL}, \mathrm{P}=0.09$, for hypertensive $v s$ normotensive, respectively) and its levels correlated significantly with anthropometric variables but not with blood pressure. Logistic regression analysis indicated that age and waist were independently associated with hypertension but not with homeostasis model assessment of insulin resistance or leptin levels. The lack of an independent association of hypertension with metabolic parameters (2-h glucose, C-reactive protein and non-HDL cholesterol) after adjustment for central adiposity suggested that visceral fat deposition may be the common mediator of the disturbances of the metabolic syndrome. Our data indicate that age and waist are major determinants of hypertension in this population of centrally obese (waist $\geq 80 \mathrm{~cm}$ ) Japanese-Brazilian women, but do not support a role for leptin in the elevation of blood pressure.
\end{abstract}

Key words

- Hypertension

- Body fat distribution

- Insulin

- Leptin

- Centrally obese

- Metabolic syndrome

\footnotetext{
* Members of the Japanese-Brazilian Diabetes Study Group: K. Osiro, M. lunes (in memoriam), S.R.G. Ferreira, S.G.A. Gimeno (Departamento de Medicina Preventiva, Universidade Federal de São Paulo, São Paulo, SP, Brasil); L.K. Matsumura, R.C.S. Moises (Disciplina de Endocrinologia, Departamento de Clínica Médica, Universidade Federal de São Paulo, São Paulo, SP, Brasil); N. Barros Junior (Departamento de Cirurgia, Universidade Federal de São Paulo, São Paulo, SP, Brasil); A.T. Hirai, B. Almeida, R.D. Freire (Programa de Pós-graduação, Endocrinologia Clínica, Universidade Federal de São Paulo, São Paulo, SP, Brasil); L.J. Franco (Departamento de Medicina Preventiva, Faculdade de Medicina de Ribeirão Preto, Universidade de São Paulo, Ribeirão Preto, SP, Brasil); M. Kikuchi (Universidade de São Paulo, São Paulo, SP, Brasil); M.A. Cardoso (Departamento de Epidemiologia e Saúde Pública, Universidade Estadual de São Paulo, São Paulo, SP, Brasil); N. Tomita (Faculdade de Odontologia, Universidade de São Paulo, Bauru, SP, Brasil); R. Chaim (Faculdade de Nutrição, Universidade Sagrado Coração, Bauru, SP, Brasil); K. Wakisaka (Centro de Estudo NipoBrasileiro, São Paulo, SP, Brasil).
} 


\section{Introduction}

Several metabolic disturbances found in conditions of excessive adiposity are associated with hypertension. A close relationship between body adiposity and insulin and leptin levels has been reported (1) but it is not known whether or not the latter contributes independently to elevation of blood pressure. Schutte et al. (2) found that leptin was directly associated with blood pressure and negatively associated with arterial compliance in obese African women with hypertension. In addition to the action of this adipocyte-derived hormone on satiety through hypothalamic receptors, leptin seems to increase sympathetic nervous system (SNS) activity (3). Based on the importance of sympathetic tone in the pathophysiology of hypertension, a role for leptin has been suggested as the link between obesity and hypertension (4).

Data obtained in obese hyperleptinemic agouti yellow mice have indicated selective resistance to the action of leptin on the hypothalamus; the maintenance of SNS hyperactivity in these animals could explain blood pressure elevation (5). Similarly, the hyperleptinemia found in human obesity also suggests a leptin resistance state (6), but it is an intriguing fact that leptin is more closely related to peripheral fat mass (subcutaneous) while hypertension-related metabolic disturbances are mainly associated with visceral fat.

Literature data are controversial since some studies have found no correlation between leptinemia and hypertension (7) and others have found a positive association between hypertension and leptinemia in women, but all of them included obese subjects $(2,8,9)$. An epidemiological study on the prevalence of diabetes and associated diseases in Japanese-Brazilians showed that such a population is at high risk for the metabolic syndrome including hypertension. Interestingly, their body mass index (BMI) is not compatible with obesity according to the values proposed by the WHO for Caucasians, despite showing centrally distributed adiposity (10). Therefore, Japanese-Brazilians constitute an interesting model that could permit an independent analysis of the effects of central fat on the development of metabolic syndrome. It would be interesting to determine whether visceral fat is more relevant than subcutaneous fat to the elevation of blood pressure and metabolic disturbances.

The present study assessed the relationships of hormonal variables with hypertension in Japanese-Brazilian women, adjusted for BMI or waist circumference. Specifically, independent associations of insulin and leptin levels with elevated blood pressure were investigated.

\section{Patients and Methods}

\section{Study population}

A survey conducted on a Japanese-Brazilian population living in Bauru, SP, Brazil, was designed to estimate the prevalence of diabetes and associated diseases (11). The entire population over 30 years of age $(\mathrm{N}=$ 1,751) was invited to participate. Clinical and laboratory data from 1,330 first- (born in Japan) and second-generation (born in Brazil) subjects were collected. Among 717 women, 233 had diabetes.

The study was approved by the Ethics Committee of the Universidade Federal de São Paulo. After giving written informed consent, participants were interviewed at home using standardized questionnaires and scheduled for clinical and laboratory procedures after an overnight fast.

\section{Protocol}

BMI was calculated as weight (kilograms) divided by height (meters) squared and waist circumference was measured at the umbilicus. Instead of a BMI cutoff of $30 \mathrm{~kg} / \mathrm{m}^{2}$ (12) 
to define obesity, the value of $25.0 \mathrm{~kg} / \mathrm{m}^{2}$ was employed in the present study, as previously proposed for Japanese populations (13). Women with a waist circumference $\geq 80 \mathrm{~cm}$ were characterized as centrally obese (13). Bioelectrical impedance was used to assess body composition and fat mass was expressed as percentage. Blood pressure was measured three times after a 10-min rest in the sitting position with an automatic device (Omron model HEM-712C, Omron Health Care, Inc., Vernon Hills, IL, USA). Hypertension was defined as systolic or diastolic blood pressure $\geq 140 / 90 \mathrm{mmHg}$ or the use of antihypertensive medications (14). Blood samples were obtained twice, i.e., under fasting conditions and $2 \mathrm{~h}$ after the ingestion of $75 \mathrm{~g}$ glucose. The glucose load was administered to non-diabetic subjects and to those with self-reported diabetes mellitus but fasting capillary glucose $<11.1 \mathrm{mmol} / \mathrm{L}$, screened by the use of glucose-oxidase strips. Glucose tolerance status was based on 1999 WHO criteria (15) or use of antidiabetic agents. Considering the heterogeneity of $\beta$ cell secretory capacity and circulating insulin levels, diabetic subjects (fasting plasma glucose $\geq 7 \mathrm{mmol} / \mathrm{L}$ or 2 -h level $\geq 11.1 \mathrm{mmol} /$ L) were excluded from the analysis, which was carried out on 484 nondiabetic women.

Plasma glucose and uric acid were determined by routine methods. Cholesterol contents of lipoprotein fractions and triglycerides were measured enzymatically and processed with an automatic analyzer. C-reactive protein was determined by a chemiluminescent immunometric assay (normal range: $0.05-0.11 \mathrm{mg} / \mathrm{dL}$ ). Sera were stored at $-20^{\circ} \mathrm{C}$ for hormonal determinations. Insulin was determined by a monoclonal antibody-based immunofluorimetric assay (AutoDelfia, Perkin-Elmer Life Sciences Inc., Norton, $\mathrm{OH}$, USA) and leptin by radioimmunoassay (Linco Research Inc., St. Charles, MO, USA). Insulin sensitivity was assessed by the homeostasis model assessment of insulin resistance (HOMA-IR) according to Matthews et al. (16).

Statistical analysis was performed using the Stata 7.0 software package (Stata Corporation, College Station, TX, USA). Data from the normo- and hypertensive groups were compared by the unpaired Student $t$-test or Wilcoxon test according to Levene's test for equality of variances. Data are reported as means \pm SD or median and range. Prevalence rates were estimated by point and $95 \%$ confidence interval $(95 \% \mathrm{CI})$. The chi-square test was used to compare frequencies. Pearson coefficient was used to test the correlation of blood pressure and leptin with demographic, clinical and laboratory parameters. Backward models of logistic regression were created including hypertension as the dependent variable and leptin as the main exposure of interest, adjusted for a number of other variables (age, waist, BMI, fat mass, fasting and 2-h glucose, 2-h insulin, HOMAIR, uric acid, C-reactive protein, serum creatinine, and lipid profile). The level of significance was set at $\mathrm{P}<0.05$ in all analyses.

\section{Results}

Among the 484 nondiabetic JapaneseBrazilian women studied, contrasting prevalence rates of obesity were observed when different diagnostic criteria were used. Using WHO criteria (12), 5.6\% obese subjects were found. However, this rate increased to $32.0 \%$ when specific criteria for Asians (13) were applied. Also, a high $37.8 \%$ prevalence of central adiposity was observed.

Patients were stratified according to the presence of hypertension, whose prevalence rate was 33\% (95\% CI: 29-38\%). As expected, the women in the hypertensive group $(\mathrm{N}=162)$ were older and showed an unfavorable metabolic profile and higher BMI than the normotensive group (Table 1). Mean insulin levels were higher in hypertensive than in normotensive subjects $(8.0 \pm 6.2 \mathrm{vs}$ $7.1 \pm 4.9 \mu \mathrm{U} / \mathrm{mL}, \mathrm{P}<0.05)$ but the difference in leptin levels was not statistically signifi- 
cant $(8.2 \pm 6.8$ vs $7.2 \pm 6.6 \mathrm{ng} / \mathrm{mL}, \mathrm{P}=$ 0.092).

Blood pressure correlated significantly with anthropometric parameters and also with insulin levels despite a low correlation coefficient (Table 2). On the other hand, no correlation was detected between blood pressure and leptin levels. The final model of

Table 1. Main characteristics of nondiabetic Japanese-Brazilians according to the presence of hypertension.

$$
\text { Normotensive }(\mathrm{N}=322) \quad \text { Hypertensive }(\mathrm{N}=162)
$$

\begin{tabular}{lcr}
\hline Age (years) & $52 \pm 12$ & $61 \pm 11^{*}$ \\
Systolic BP (mmHg) & $114 \pm 14.0$ & $151 \pm 20.6^{*}$ \\
Diastolic BP (mmHg) & $69 \pm 8.5$ & $86 \pm 12.3^{*}$ \\
BMI $\left(\mathrm{kg} / \mathrm{m}^{2}\right)$ & $23.3 \pm 3.4$ & $24.9 \pm 4.2^{*}$ \\
Waist circumference (cm) & $76.3 \pm 8.2$ & $81.1 \pm 10.1^{*}$ \\
Fat mass $(\%)$ & $28 \pm 6$ & $29 \pm 6^{*}$ \\
Fasting glucose (mmol/L) & $5.9 \pm 0.5$ & $6.0 \pm 0.5^{*}$ \\
2-h glucose (mmol/L) & $7.1 \pm 1.5$ & $7.6 \pm 1.4^{*}$ \\
Total cholesterol $(\mathrm{mmol} / \mathrm{L})$ & $5.3 \pm 1.2$ & $6.7 \pm 1.1^{*}$ \\
HDL-cholesterol $(\mathrm{mmol} / \mathrm{L})$ & $1.3 \pm 0.3$ & $1.4 \pm 0.2$ \\
LDL-cholesterol $(\mathrm{mmol} / \mathrm{L})$ & $3.3 \pm 0.9$ & $3.6 \pm 0.9^{*}$ \\
Triglyceride $(\mathrm{mmol} / \mathrm{L})$ & $1.6 \pm 1.1$ & $2.1 \pm 1.4^{*}$ \\
Uric acid $(\mathrm{mmol} / \mathrm{L})$ & $0.291 \pm 0.101$ & $0.327 \pm 0.071^{*}$ \\
C-reactive protein $(\mathrm{mg} / \mathrm{dL})$ & $0.200 \pm 0.57$ & $0.250 \pm 0.29$ \\
Fasting insulin $(\mu \mathrm{U} / \mathrm{mL})$ & $7.1 \pm 4.9$ & $8.0 \pm 6.2$ \\
2-h insulin $(\mu \mathrm{U} / \mathrm{mL})$ & $52.6 \pm 45.4$ & $67.9 \pm 55.9^{*}$ \\
HOMA-IR & $1.9 \pm 1.3$ & $2.2 \pm 1.8$ \\
Leptin $(\mathrm{ng} / \mathrm{mL})$ & $7.2 \pm 6.6$ & $8.2 \pm 6.8$ \\
\end{tabular}

Data are reported as mean $\pm \mathrm{SD}$. Reference values: total cholesterol - desirable: $<5.2$ $\mathrm{mmol} / \mathrm{L}$; borderline: $5.2-6.1 \mathrm{mmol} / \mathrm{L}$; high risk: $>6.2 \mathrm{mmol} / \mathrm{L}$. HDL-c: $0.88-2.28 \mathrm{mmol} / \mathrm{L}$. LDL-C: $<3.37 \mathrm{mmol} / \mathrm{L}$. Triglyceride: $<1.65 \mathrm{~g} / \mathrm{L}$. Fasting insulin: $6-35 \mu \mathrm{U} / \mathrm{mL}$. Leptin (women with BMI 18-25 kg/m²): $7.4 \pm 3.7 \mathrm{ng} / \mathrm{mL}$. BP = blood pressure; $\mathrm{BMI}=$ body mass index; HOMA-IR = homeostasis model assessment of insulin resistance. ${ }^{*} \mathrm{P}<0.05$ compared to normotensive subjects (Student $t$-test or Wilcoxon test according to Levene's test for equality of variances).

Table 2. Correlation coefficients between variables of main interest.

\begin{tabular}{llllllll}
\hline & Mean BP & BMI & Waist & Fat mass & 2-h insulin & Leptin & HOMA-IR \\
\hline Mean BP & 1.00 & & & & & & \\
BMI & $0.24^{*}$ & 1.00 & & & & & \\
Waist & $0.29^{*}$ & $0.84^{*}$ & 1.00 & & & & \\
Fat mass & $0.18^{*}$ & $0.79^{*}$ & $0.71^{*}$ & 1.00 & & & \\
2-h insulin & $0.09^{*}$ & $0.30^{*}$ & $0.30^{*}$ & $0.23^{*}$ & 1.00 & & \\
Leptin & 0.01 & $0.14^{*}$ & $0.14^{*}$ & $0.11^{*}$ & $0.15^{*}$ & 1.00 & \\
HOMA-IR & $0.10^{*}$ & $0.32^{*}$ & $0.31^{*}$ & $0.23^{*}$ & $0.99^{*}$ & 0.09 & 1.00
\end{tabular}

${ }^{*} \mathrm{P}<0.05$, correlation coefficients by the Pearson method. $\mathrm{BP}=$ blood pressure; $\mathrm{BMI}=$ body mass index; HOMA-IR = homeostasis model assessment of insulin resistance. logistic regression analysis is depicted in Table 3. Fasting insulin and plasma glucose or HOMA-IR alternatively entered the models; the same procedure was adopted for BMI or waist circumference, providing similar results (data not shown). Age, waist and uric acid were independently associated with hypertension after adjusting for 2-h glucose, C-reactive protein and non-HDL cholesterol. A borderline significance was detected for the association of hypertension with triglycerides $(P=0.07)$, but not with leptin or HOMA-IR.

\section{Discussion}

Hypertension - as a component of the metabolic syndrome - results from complex and multifactorial mechanisms including obesity-induced metabolic and hormonal disturbances. Among these mechanisms, adipose tissue-derived molecules have direct (non-esterified fatty acids, angiotensin II, nitric oxide, prostacyclin) or indirect (leptin) effects on the regulation of SNS activity, sodium reabsorption and renin-angiotensin-aldosterone system activity (17). The association of hypertension with hyperinsulinemia was documented in some (18) but not all studies (19). Controversies also exist regarding an association between hypertension and leptin levels (20-27).

An association between hyperleptinemia and hypertension has been found in obese subjects at risk for metabolic syndrome (2,8,27-29). Most studies regarding the involvement of hormonal factors in the genesis of hypertension include obese subjects. Japanese-Brazilians offer an opportunity to more specifically investigate the importance of body fat distribution for blood pressure elevation, since they constitute a population with localized central adiposity without overall obesity and a high prevalence of metabolic syndrome (9). The mean BMI of this population would not be considered elevated according to WHO criteria. However, using 
specific criteria for Asians (13), high prevalence rates of obesity and central adiposity were observed. A stronger correlation with waist than with fat mass may indicate the importance of the visceral component for the BMI. Our finding of low leptin levels was expected considering their BMI and fat mass which, together with gender, explain $81 \%$ of leptin variance in humans (30).

Studies in animals and humans have suggested a link between leptin and hypertension by activation of the SNS and an increase of sodium reabsorption $(2,3)$. Hyperleptinemia was found in obese rats with increased blood pressure (20) and hormone administration induced sympathetic hyperactivity $(21,22)$. These data were in agreement with a predominant pressor action induced by peripheral or central infusion of leptin $(23,24)$ but not with the finding of increased endothelial nitric oxide in isolated blood vessels (25) or the results of acute infusion which suggested a depressor action (26).

Leptin - mainly secreted by the subcutaneous adipose tissue - was used to indirectly investigate the possible influence of total fat on blood pressure in the present study. The lack of association of this hormone with hypertension in logistic regression did not support a role for blood pressure elevation, which should be investigated in a longitudinal study.

In this context, some studies have not found an independent association of leptin with blood pressure when adjusted for general obesity. El-Gharbawy et al. (31) found a correlation between leptin and blood pressure in African women with hypertension and obesity, which disappeared after adjustments for other components of the insulin resistance syndrome. In a similar population, Schutte et al. (2) found that leptin was positively associated with systolic blood pressure in obese but not in lean women. These findings agree with others, which showed that angiotensin II and leptin levels were strong predictors of blood pressure in obese women, but not in lean women in whom only the former predicted blood pressure (32). Also, in a Chinese population, Hu et al. (33) observed that a significant association between leptin and blood pressure disappeared after adjusting for BMI, whereas the association between BMI and blood pressure persisted after adjusting for leptin levels.

Considering the effect of hyperinsulinemia in increasing the expression of the ob gene in adipocytes (34), it is probable that previously reported associations of leptin with blood pressure were due to the influence of concomitant elevation of insulin levels in obese subjects. In fact, we confirmed this significant correlation between these hormone levels, in agreement with other studies conducted in non-diabetic subjects (35) and in Japanese diabetic subjects with normal BMI (34).

Our final logistic regression model showed that waist circumference - indicative of visceral fat deposition - was independently associated with hypertension. This is in agreement with the reported differences in the prevalence of hypertension according to body fat distribution (36). Prospective studies indicated that visceral fat was implicated in metabolic and hemodynamic abnormalities (35) linked to insulin resistance. Activation of the SNS may be one of the mechanisms underlying such abnormalities. In the present study, weak correlation coefficients for

Table 3. Final model of logistic regression (hypertension as the dependent variable).

\begin{tabular}{lcccc}
\hline & OR & $P$ & \multicolumn{2}{c}{$95 \% \mathrm{Cl}$} \\
\hline Age & 1.011 & 0.000 & 1.008 & 1.015 \\
Waist & 1.007 & 0.028 & 1.001 & 1.011 \\
Uric acid & 1.043 & 0.038 & 1.002 & 1.084 \\
Triglyceride & 1.000 & 0.077 & 1.000 & 1.001 \\
HOMA-IR & 1.010 & 0.507 & -1.020 & 1.041 \\
Leptin & -1.002 & 0.648 & -1.008 & 1.005
\end{tabular}

Adjusted for 2-h glucose, C-reactive protein and non-HDL cholesterol (nonsignificant). HOMA-IR = homeostasis model assessment of insulin resistance; OR = odds ratio; $95 \% \mathrm{Cl}=95 \%$ confidence interval. 
blood pressure with 2-h insulin and HOMAIR were found but not confirmed in multivariate analysis. Adjustments for age and waist may have weakened such association. In fact, Rocchini et al. (19) suggested that SNS activation is important in the pathogenesis of obesity hypertension, whereas insulin resistance associated with obesity might be mediated in part by alterations in the central or peripheral $\alpha$-adrenergic receptors, and concluded that obesity-induced hypertension and obesity-induced insulin resistance are not directly related (19). In addition, it is known that a simultaneous impairment of vascular resistance and arterial compliance is associated with aging in different patterns of hypertension (37).

Our data support the hypothesis that cen- tral distribution of fat should be a determinant of hypertension. In the presence of increased central adiposity, concomitant with low total adiposity, typical associations of metabolic parameters with hypertension are minimized. A role of leptin in blood pressure elevation in this specific population of Japanese-Brazilian women was not suggested, in agreement with the hypothesis that visceral fat-related factors should be more relevant than subcutaneous fat in the association with hypertension.

\section{Acknowledgments}

We are indebted to the participants in the Japanese-Brazilian Diabetes Study for their continuing outstanding cooperation.

\section{References}

1. Courten M, Zimmet P, Hodge A et al. (1997). Hyperleptinemia: the missing link in the metabolic syndrome. Diabetic Medicine, 14: 200208.

2. Schutte R, Huisman HW, Schtte AE et al. (2005). Leptin is independently associated with systolic blood pressure, pulse pressure and arterial compliance in hypertensive African women with increased adiposity: the POWIRS study. Journal of Human Hypertension, 19: 535-541.

3. Collins S, Kuhn CM, Pettro AE et al. (1996). Role of leptin in fat regulation. Nature, 380: 677.

4. Hall JE, Hildebrandt DA, Kuo J et al. (2000). Role of sympathetic nervous system and neuropeptides in obesity hypertension. Brazilian Journal of Medical and Biological Research, 33: 605-618.

5. Correia ML, Haynes WG, Rahmouni K et al. (2000). The concept of selective leptin resistance. Evidence from Agouti yellow obese mice. Diabetes, 51: 439-442.

6. Flier JS (1998). What is in a name? In search of leptin's physiologic role. Journal of Clinical Endocrinology and Metabolism, 83: 14071413.

7. Mohamed-Ali V, Pinkney JH, Panahloo A et al. (1997). Relationships between plasma leptin and insulin concentration, but not insulin resistance, in non-insulin-dependent (type 2) diabetes mellitus. Diabetic Medicine, 14: 376-380.

8. Adamczak M, Kokot F \& Wiecek A (2000). Relationship between plasma rennin profile and leptinaemia in patients with essential hypertension. Journal of Human Hypertension, 14: 503-509.

9. Corica F, Corsonello A, lentile R et al. (2001). Leptin and norepinephrine plasma concentrations during glucose loading in normotensive and hypertensive obese women. American Journal of $\mathrm{Hy}$ pertension, 14: 619-626.

10. Ferreira SRG, Lerario DDG, Gimeno SGA et al. (2002). Obesity and central adiposity in Japanese immigrants: role of the Western dietary pattern. Journal of Epidemiology, 12: 431-438.

11. Gimeno SGA, Ferreira SRG, Franco LJ et al. (2002). Prevalence and 7-year incidence of type 2 diabetes mellitus in a JapaneseBrazilian population: an alarming public health problem. Diabetologia, 45: 1635-1638.

12. World Health Organization (1998). Obesity: preventing and managing the global epidemics. Report of WHO Consultation on Obesity. World Health Organization, Geneva, Switzerland.

13. World Health Organization - WHO (2000). Steering Committee of the Western Pacific Region of the WHO, the International Association for the Study of Obesity Task Force. The Asia-Pacific perspective: redefining obesity and its treatment. World Health Organization, Geneva, Switzerland.

14. Anonymous (1997). The Sixth Report of the Joint National Committee on Prevention, Detection, Evaluation, and Treatment of High Blood Pressure. Archives of Internal Medicine, 157: 2413-2440.

15. World Health Organization (1999). Definition, diagnosis and classification of diabetes mellitus and its complications. Department of Noncommunicable Disease Surveillance, Geneva, Switzerland.

16. Matthews DR, Hosker JP, Rudenski AS et al. (1985). Homeostasis model assessment: insulin resistance and B-cell function from fasting plasma glucose and insulin concentrations in man. Diabetologia, 28: 412-419.

17. Engeli S \& Sharma AM (2000). Role of adipose tissue for cardiovascular-renal regulation in health and disease. Hormone and Metabolic Research, 32: 485-499.

18. Mark AL, Correia M, Morgan DA et al. (1999). Obesity-induced hypertension. New concepts from the emerging biology of obesity. Hypertension, 33 (Part II): 537-541.

19. Rocchini AP, Yang JQ \& Gokee A (2004). Hypertension and insulin 
resistance are not directly related in obese dogs. Hypertension, 43: 1011-1016.

20. Jens $\mathrm{HH}$, Jens JH, Soren M et al. (2000). Elevated circulating leptin levels in arterial hypertension: relationship to arteriovenous overflow and extraction of leptin. Clinical Science, 99: 527-534.

21. Correia MLG, Morgan DA, Mitchell JL et al. (2001). Role of corticotropin-releasing factor in effects of leptin on sympathetic nerve activity and arterial pressure. Hypertension, 38: 384-388.

22. Correia MLG, Morgan DA, Sivitz WI et al. (2002). Hemodynamic consequences of neuropeptide $\mathrm{Y}$-induced obesity. American Journal of Hypertension, 15: 137-142.

23. Correia MLG, Morgan DA, Sivitz WI et al. (2001). Leptin acts in the central nervous system to produce dose-dependent changes in arterial pressure. Hypertension, 37: 936-942.

24. Shek EW, Brands MW \& Hall JE (1998). Chronic leptin infusion increases arterial pressure. Hypertension, 31 (Part 2): 409-414.

25. Zhang R \& Reisin E (2000). Obesity-hypertension: the effects on cardiovascular and renal systems. American Journal of Hypertension, 13: 1308-1314.

26. Mitchell JL, Correia MLG, Morgan DA et al. (2001). Does leptin stimulate nitric oxide to oppose the effects of sympathetic activation? Hypertension, 38: 1081-1086.

27. Sudi K, Gallistl S, Trobinger M et al. (2000). Insulin and insulin resistance index are not independent determinants for the variation in leptin in obese children and adolescents. Journal of Pediatric Endocrinology and Metabolism, 13: 923-933.

28. Kunz I, Schorr U, Klaus S et al. (2000). Resting metabolic rate and substrate use in obesity hypertension. Hypertension, 36: 26-32.

29. Laivuori $\mathrm{H}$, Kaaja $\mathrm{R}$, Koistinen $\mathrm{H}$ et al. (2000). Leptin during and after preeclamptic or normal pregnancy: its relation to serum insulin and insulin sensitivity. Metabolism, 49: 259-263.

30. Kennedy A, Gettys TW, Watson P et al. (1997). The metabolic significance of leptin in humans: gender-based differences in relationship to adiposity, insulin sensitivity, and energy expenditure. Journal of Clinical Endocrinology and Metabolism, 82: 1293-1300.

31. El-Gharbawy AH, Kotchen JM, Grim CE et al. (2002). Genderspecific correlates of leptin with hypertension-related phenotypes in African Americans. American Journal of Hypertension, 15: 989-993.

32. Hazimi-Al AM \& Syiamic AY (2004). Relationship between plasma angiotensin II, leptin and arterial blood pressure. Saudi Medical Journal, 25: 1193-1198.

33. Hu FB, Chen C, Wang B et al. (2001). Leptin concentrations in relation to overall adiposity, fat distribution, and blood pressure in a rural Chinese population. International Journal of Obesity, 25: 121 125.

34. Asakawa H, Tokunaga K \& Kawakami F (2001). Relationship of leptin level with metabolic disorders and hypertension in Japanese type 2 diabetes mellitus patients. Journal of Diabetes and its Complications, 15: 57-62.

35. Haffner SM, Mykkanen L, Rainwater DL et al. (1999). Is leptin concentration associated with the insulin resistance syndrome in nondiabetic men? Obesity Research, 7: 164-169.

36. Larsson B, Svardsudd K, Welin L et al. (1984). Abdominal adipose tissue distribution, obesity, and risk of cardiovascular disease and death: 13 year follow-up of participants in the study of men born in 1913. British Medical Journal, 288: 1401-1404.

37. Taddei S, Virdis A, Ghiadoni L et al. (2001). Age-related reduction of NO availability and oxidative stress in humans. Hypertension, 38 . 274-279. 